\title{
Quantum vortex dynamics in two-dimensional neutral superfluids
}

\author{
C.-C. Joseph Wang,,$^{1, *}$ R. A. Duine, ${ }^{2, \dagger}$ and A. H. MacDonald ${ }^{2, \ddagger}$ \\ ${ }^{1}$ The University of Texas at Austin, Department of Physics, 1 University Station C1600, Austin, Texas 78712-0264 \\ ${ }^{2}$ Institute for Theoretical Physics, Utrecht University, Leuvenlaan 4, NL-3584 CE Utrecht, The Netherlands
}

(Received 1 October 2009; published 12 January 2010)

\begin{abstract}
We derive an effective action for the vortex-position degree of freedom in a superfluid by integrating out condensate phase- and density-fluctuation environmental modes. When the quantum dynamics of environmental fluctuations is neglected, we confirm the occurrence of the vortex Magnus force and obtain an expression for the vortex mass. We find that this adiabatic approximation is valid only when the superfluid droplet radius $R$, or the typical distance between vortices, is very much larger than the coherence length $\xi$. We go beyond the adiabatic approximation numerically, accounting for the quantum dynamics of environmental modes and capturing their dissipative coupling to condensate dynamics. For the case of an optical-lattice superfluid, we demonstrate that vortex motion damping can be adjusted by tuning the ratio between the tunneling energy $J$ and the on-site interaction energy $U$. We comment on the possibility of realizing vortex-Landau-level physics.
\end{abstract}

DOI: 10.1103/PhysRevA.81.013609

PACS number(s): 03.75.Kk, 47.32.C-, 47.37.+q

\section{INTRODUCTION}

Superfluids accommodate angular momentum by nucleating quantized vortices [1,2]. The rapid progress of research on Bose-Einstein condensed, ultracold atoms in magnetic traps [3-6] and optical lattices [7,8] over the past decade has opened up an opportunity to study vortex physics in a new setting [9-12]. New possibilities for exploring phenomena in which the quantum behavior of vortices is important are particularly attractive. It is known, for instance, that quantum fluctuations induce vortex-lattice melting [13] when the number of atoms per vortex is less than around ten and that boson quantum Hall states [14] (in which vortices form incompressible quantum fluids) emerge at still lower atom densities (for a recent review, consult Ref. [15]). Phase separation between different vortex states driven by inhomogeneity in the condensate density [16] and vortex-Peierls states in some optical lattices are manifestations of quantumness in vortex physics that is enhanced by geometrical frustration [17].

In spite of the long history of vortex physics in neutral superfluids, there are still some controversies surrounding the understanding of vortex quantum dynamics [18]. The main concerns center on the sources of forces on a quantum vortex and on the effective vortex mass in various circumstances [19-22]. The consensus view (for completeness, however, see also Ref. [23]), based on either Berry-phase arguments [24] or hydrodynamic theory [25], is that a vortex has a finite mass and experiences a Magnus force proportional to the boson density in its vicinity. According to these theories then, a vortex behaves like a massive charged particle in an effective magnetic field due to the background bosons [26,27]. Vortex kinetic energy should therefore be subject to the same Landau quantization conditions which qualitatively

\footnotetext{
*joseph@physics.georgetown.edu, joseph@physics.utexas.edu; URL:http://www.ph.utexas.edu/ joseph/joseph.html

${ }^{\dagger}$ R.A.Duine@uu.nl; URL:http://www.phys.uu.nl/ duine

${ }^{\ddagger}$ macd@physics.utexas.edu; URL:http://www.ph.utexas.edu/ $\sim$ macdgrp
}

alter the properties of electrons subject to an external magnetic field. Vortex-Landau quantization has nevertheless never been observed. A key aim of this work is to help point the way toward circumstances in which this distinctive aspect of vortex quantum mechanics is more likely to be exhibited.

In early work on the properties of vortices, the vortex mass $m_{v}$ was often treated as a purely phenomenological parameter [28]. More attention has been given to the microscopic underpinning of vortex mass in recent work. For example, Duan [29] demonstrated that the vortex mass is generated by the superfluid compressibility, although the Magnus force was not considered. Arovas and Freire [30] formulated a theory of vortex mass by assuming that the coupling between the vortex position and environment is dictated by gauge invariance as in electromagnetism, with the vortex playing the role of the charged particle and phonons that of the electromagnetic field. In their approach, the resulting minimal coupling leads to phonon emission by a moving vortex, which gives the vortex a frequency-dependent effective mass. Further progress in clarifying the microscopic physics of the vortex mass was achieved more recently in Ref. [31], which approached the issue by using perturbation theory with a rotating pinning potential. These authors confirmed that the vortex mass is due to density fluctuations in the superfluid and showed that the value of the mass can depend on details of the vortex-core structure.

In this article, we use a different approach to derive an effective action for an isolated vortex. We start with a careful identification of the vortex-translation zero modes in the Gaussian fluctuation spectrum of a superfluid which contains a vortex. We then integrate out all the remaining Gaussian fluctuations, which consist of superfluid density and phase fluctuations distorted by the presence of the vortex, to obtain an effective theory which depends only on the vortex-position degree of freedom. In the adiabatic limit, in which the quantum dynamics of the environmental fluctuations can be ignored, we reproduce the vortex mass predicted by Duan [29]. We confirm that the appearance of the vortex mass is due primarily to the coupling between vortices and condensate-density fluctuations outside the vortex core. When quantum fluctuations of the 
environment modes are allowed, we find that the picture in which the vortex exists as a well-defined quantum particle with an energy-independent mass holds only when the vortices are in a in superfluid cloud with radius $R$, very much larger than the coherence length $\xi$. In a system with many vortices, the corresponding condition requires that the typical distance between vortices be very much larger than $\xi$.

We consider, in addition, the situation of a vortex in an optical-lattice condensate and calculate the vortex mass and effective magnetic field numerically. Numerical results for the vortex mass tend to agree reasonably well with analytical results based on a model.

Numerical results for the effective magnetic field, however, depend strongly on the optical lattice and do not compare favorably with analytical results. For large vortex-core sizes the discrepancy may be explained as a consequence of the large fraction of the system area that is occupied by vortex cores. For small vortex cores, on the other hand, lattice pinning by the vortex core plays an important role. Taken together, these two effects imply that sharp vortex cyclotron resonances and vortex-Landau-level physics are difficult to observe in optical-lattice Bose condensates.

The remainder of this article is organized as follows. In Sec. II we outline our model and present our approach to the evaluation of the vortex mass and effective magnetic field. We develop this approach analytically for the continuum limit. In Sec. III we present results of numerical calculations for lattice bosons. We end with conclusions in Sec. IV.

\section{THEORETICAL FORMULATION}

\section{A. Effective action}

We consider a dilute interacting bosonic gas in a reduced two-dimensional geometry. Our starting point is the partition function for the two-dimensional dilute gas with contact interaction $g>0$, written as a coherent-state path integral over the fields $\phi^{*}(\mathbf{x}, \tau)$ and $\phi(\mathbf{x}, \tau)$. It is given by

$$
Z=\int d\left[\phi^{*}\right] d[\phi] e^{-S\left[\phi^{*}, \phi\right] / \hbar},
$$

with the Euclidean action $S\left[\phi^{*}, \phi\right]=\int_{0}^{\hbar \beta} d \tau L$ and Lagrangian

$$
\begin{aligned}
L\left[\phi^{*}, \phi\right]= & \int d \mathbf{x} \phi^{*}(\mathbf{x}, \tau) \\
& \times\left[\hbar \frac{\partial}{\partial \tau}-\frac{\hbar^{2} \nabla^{2}}{2 m}-\mu+\frac{g|\phi(\mathbf{x}, \tau)|^{2}}{2}\right] \phi(\mathbf{x}, \tau) \\
= & \int d \mathbf{x}\left[\phi^{*}(\mathbf{x}, \tau) \hbar \frac{\partial}{\partial \tau} \phi(\mathbf{x}, \tau)+\mathcal{E}[\phi(\mathbf{x})]\right]
\end{aligned}
$$

The functional integration in Eq. (1) is over all fields periodic on the imaginary-time axis between $\tau=0$ and $\tau=\hbar \beta \equiv$ $\hbar / k_{B} T$, where $k_{B} T$ is the thermal energy. The integral in Eq. (2) is over two-dimensional position $\mathbf{x}$. In the context of cold atoms, the model can be realized with a sufficiently strong harmonic trapping potential with frequency $\omega_{z}$ in the $z$ direction. The effective contact-interaction strength $g$ is related to the $s$-wave scattering length $a$ of the atoms by $g=4 \pi a \hbar^{2} /\left(m d_{z}\right)$, with $m$ the mass of an atom. The extent of the system in the $z$ direction is denoted by $d_{z}$ and is related to the strong harmonic trapping potential in that direction given by $d_{z} \sim \sqrt{\hbar / m \omega_{z}}$. We ignore the effects of the radial trapping potentials for now in order to avoid unessential complications.

We consider field configurations close to that of a classical vortex with counterclockwise phase winding $+2 \pi$ located at $\mathbf{r}_{v}$ :

$$
\phi(\mathbf{x}, \tau)=\phi_{v}\left[\mathbf{x}-\mathbf{r}_{v}(\tau)\right]+\delta \phi(\mathbf{x}, \tau) .
$$

Here, $\phi_{v}\left(\mathbf{x}-\mathbf{r}_{v}\right)$ is a local minimum of the energy functional $\mathcal{E}[\phi(\mathbf{x})]$ in Eq. (2) for any value of the vortex position $\mathbf{r}_{v}$. Equivalently, we can say that $\phi_{v}(\mathbf{x})$ is the stationary solution of the Euler-Lagrange equation of the action in Eq. (2) (i.e., $\delta S\left[\phi_{v}^{*}, \phi_{v}\right] / \delta \phi^{*}=0$ ) containing one vortex at the origin. Vortex translation in time is described by $\mathbf{r}_{v}(\tau)$. However, the fluctuating part of the field $\delta \phi(\mathbf{x}, \tau)$ contains zero modes that also describe the translation of the vortex. For example, a fluctuation $\delta \phi \propto \partial_{x} \phi_{v}$ describes translation of the vortex in the $x$ direction. Generally, we denote the zero modes responsible for translation of the vortex in the $x$ and $y$ directions by $\phi_{0 i}$, with $i \in\{x, y\}$. To avoid double counting in the functional integral, we have to enforce the constraint that the fluctuations $\left(\delta \phi^{*}, \delta \phi\right)$ are orthogonal to the zero modes. Hence, the fluctuations are required to obey

$$
\left\langle\phi_{0 i} \mid \delta \phi\right\rangle \equiv \int d \mathbf{x} \phi_{0 i}^{*}(\mathbf{x}) \delta \phi(\mathbf{x}, \tau)=0 .
$$

This procedure precisely separates vortex position and what we will refer to as environmental fluctuations. To enforce the above constraint and, at the same time, introduce the vortex position as a dynamical variable in the function integral, we use the Fadeev-Popov procedure $[32,33]$. We rewrite the partition function as

$$
\begin{aligned}
Z= & \int d\left[\mathbf{r}_{v}\right] d\left[\phi^{*}\right] d[\phi] \delta\left(\left\langle\phi_{0 x} \mid \delta \phi\right\rangle\right) \delta\left(\left\langle\phi_{0 y} \mid \delta \phi\right\rangle\right) \\
& \times e^{-S\left[\mathbf{r}_{v}, \delta \phi^{*}, \delta \phi\right] / \hbar}
\end{aligned}
$$

and expand the action up to quadratic order in the fluctuations. The resulting actions consist of three parts, a bare vortex-motion contribution $S_{v}$, an environmental-fluctuation contribution $S_{e}$, and a coupling term $S_{c}$ :

$$
S\left[\mathbf{r}_{v}, \delta \phi^{*}, \delta \phi\right]=S_{v}\left[\mathbf{r}_{v}\right]+S_{e}\left[\delta \phi^{*}, \delta \phi\right]+S_{c}\left[\mathbf{r}_{v}, \delta \phi^{*}, \delta \phi\right] .
$$

Because $\mathcal{E}\left[\phi_{v}\left(\mathbf{x}-\mathbf{r}_{v}\right)\right]$ is independent of $\mathbf{r}_{v}$, the bare vortex contribution to the action is due entirely to the kinetic terms:

$$
\begin{aligned}
S_{v}\left[\mathbf{r}_{v}\right]= & \left\langle\phi_{\nu} \mid \hbar \partial_{\tau} \phi_{\nu}\right\rangle=\frac{1}{2}\left[\left\langle\phi_{\nu} \mid \hbar \partial_{\tau} \phi_{\nu}\right\rangle-\left\langle\hbar \partial_{\tau} \phi_{\nu} \mid \phi_{\nu}\right\rangle\right] \\
= & i \hbar\left\{\int d \mathbf{x} \operatorname{Im}\left[\frac{\partial \phi_{v}^{*}(\mathbf{x})}{\partial x} \frac{\partial \phi_{v}(\mathbf{x})}{\partial y}\right]\right\} \\
& \times \int d \tau\left[x_{v} \dot{y}_{v}-y_{v} \dot{x}_{v}\right] .
\end{aligned}
$$

The spatial integral factor in Eq. (7) is readily evaluated for a circularly symmetric vortex wave function $\phi_{v}(\mathbf{x})=$ $f(r) \exp (i \theta)$ using polar coordinates:

$$
\begin{aligned}
\int d \mathbf{x} \operatorname{Im}\left[\frac{\partial \phi_{v}^{*}(\mathbf{x})}{\partial x} \frac{\partial \phi_{v}(\mathbf{x})}{\partial y}\right] & =2 \pi \int d r f \frac{d f}{d r} \\
& =\pi f^{2}(\infty)=\pi n_{c}
\end{aligned}
$$


where $n_{c}=\left|\phi_{v}\right|^{2}$ is the condensate density in the uniform superfluid far from the vortex. As a result, we obtain

$$
S_{v}\left[\mathbf{r}_{v}\right]=i \hbar \pi n_{c} \int_{0}^{\hbar \beta} d \tau\left[\epsilon^{i j} r_{v}^{i}(\tau) \frac{d r_{v}^{j}(\tau)}{d \tau}\right]
$$

where $\epsilon^{i j}$ is the two-dimensional Levi-Civita tensor and summation over repeated Cartesian indices $i, j \in\{x, y\}$ is implied. After a Wick rotation from imaginary to real time $(\tau \longrightarrow i t)$, this contribution to the action can be identified with the magnetic potential which appears for charged particles in a magnetic field. When added to a free-particle Lagrangian it implies kinetic energy quantization and Landau level formation. The effective magnetic length $\ell \equiv(\hbar c / q B)^{1 / 2}$ corresponding to this effective magnetic potential is $\ell_{\text {eff }}=$ $\left(2 \pi n_{c}\right)^{-1 / 2}$. The effective Aharonov-Bohm phase accumulated by a vortex wave function surrounding an area $A$ is therefore $A / \ell^{2}=2 \pi N_{c}$, where $N_{c}=A n_{c}$ is the number of enclosed condensate particles. The correspondence between this phase and the phase $2 \pi N_{v}$ experienced by bosons enclosing $N_{v}$ vortices is the origin of the boson-vortex duality properties which are useful $[26,27]$ in analyzing superconductor-insulator phase transitions.

It is convenient to express the quadratic action for quantum fluctuations of the environment $S_{e}\left[\delta \phi^{*}, \delta \phi\right]$ in the form

$$
\begin{aligned}
S_{e}= & \frac{1}{2} \int_{0}^{\hbar \beta} d \tau \\
& \times \int d \mathbf{x} \delta \Psi^{\dagger}(\mathbf{x}) \cdot\left[\left(\begin{array}{cc}
\hbar \frac{\partial}{\partial \tau} & 0 \\
0 & -\hbar \frac{\partial}{\partial \tau}
\end{array}\right)+\mathcal{H}\right] \cdot \delta \Psi(\mathbf{x}),
\end{aligned}
$$

where the vector $\delta \Psi \equiv\left[\delta \phi(\mathbf{x}, \tau), \delta \phi^{*}(\mathbf{x}, \tau)\right]^{T}$ and the hermitian Hamiltonian $\mathcal{H}$

$$
\mathcal{H}=\left\{\begin{array}{cc}
-\frac{\hbar^{2} \nabla^{2}}{2 m}-\mu+2 g\left|\phi_{v}(\mathbf{x})\right|^{2} & g\left[\left(\phi_{v}(\mathbf{x})\right] 2\right. \\
g\left[\phi_{v}^{*}(\mathbf{x})\right] 2 & -\frac{\hbar^{2} \nabla^{2}}{2 m}-\mu+2 g\left|\phi_{v}(\mathbf{x})\right|^{2}
\end{array}\right\} .
$$

The action contribution that describes coupling between vortex translation and the quantum fluctuations of its condensate environment is

$S_{c}=\frac{\hbar}{2} \int_{0}^{\hbar \beta} d \tau d \mathbf{x}\left[\partial_{i} \phi_{v}^{*}(\mathbf{x}) \delta \phi(\mathbf{x}, \tau)-\delta \phi^{*}(\mathbf{x}, \tau) \partial_{i} \phi_{v}(\mathbf{x})\right] \dot{r}_{v}^{i}(\tau)$.

Only kinetic Berry-phase coupling terms appear here because the vortex-translation mode is a (zero-energy) eigenfunction of the hermitian Hamiltonian $\mathcal{H}$.

In order to focus on vortex properties, we next integrate out the environmental fluctuations $\delta \phi^{*}$ and $\delta \phi$. The constraints imposed by the $\delta$-functional in the functional integral [Eq. (5)] are conveniently applied by expanding the fluctuation fields on the basis of eigenfunctions of the Hamiltonian $\mathcal{H}$ :

$$
\chi_{\alpha} \equiv\left(\begin{array}{l}
\chi_{\alpha}^{1} \\
\chi_{\alpha}^{2}
\end{array}\right)
$$

We expect $\mathcal{H}$ to have three zero-energy eigenfunctions. The first $\chi_{0 p}=\left(\phi_{v},-\phi_{v}^{*}\right)^{T}$ is the global-phase mode that results from the $U(1)$ invariance of the action in Eq. (2). This mode makes no contribution to the dynamics of the vortex, as will be shown later. The other two easily identifiable modes on which we focus are, strictly speaking, zero modes only in the absence of vortex pinning and represent vortex translation in orthogonal directions in the $\hat{x}-\hat{y}$ plane. Hereafter, we will refer to these translational modes as zero modes in the discussion. Suitable linear combinations correspond to translations along translation along any direction in the $x-y$ plane. We choose as basis functions $\chi_{0 i \in\{x, y\}} \propto\left(\partial_{i} \phi_{v}, \partial_{i} \phi_{v}^{*}\right)^{T}$, where $i=x, y$. One can demonstrate by direct substitution that these are indeed zero-energy eigenfunctions of $\mathcal{H}$. All other fluctuations can be expanded in terms of the finite-energy $\epsilon_{\alpha}$ eigenfunctions of $\mathcal{H}$ and correspond to density and phase fluctuations of a superfluid which has been distorted by the presence of a vortex. For the purpose of later discussions, we define the quantity $\Gamma_{\alpha^{\prime} \alpha}$, an inner product between eigenfunctions $\alpha^{\prime}, \alpha$, as $\Gamma_{\alpha^{\prime} \alpha}=\frac{1}{2}\left\langle\chi_{\alpha^{\prime}}^{\dagger}\left|\sigma_{z}\right| \chi_{\alpha}\right\rangle$, where $\sigma_{z}$ is the $z$-th Pauli matrix, with the hermitian property $\Gamma_{\alpha^{\prime} \alpha}=\Gamma_{\alpha \alpha^{\prime}}^{*}$. For example, the Berry-phase coupling between the $x$ and the $y$ components of the vortex displacement, considered in Eq. (8), is $\Gamma_{x y}$, which is purely imaginary.

\section{B. Adiabatic vortex dynamics and effective mass}

Now we consider vortex dynamics in the adiabatic approximation. This amounts to neglecting the explicit time dependence of environmental modes in the action of fluctuations $S_{e}\left[\delta \phi^{*}, \delta \phi\right]$. We expand the microscopic action $S$ in the basis of fluctuation Hamiltonian $\mathcal{H}$ eigenfunctions; that is, $\left[\delta \phi(\mathbf{x}, \tau), \delta \phi^{*}(\mathbf{x}, \tau)\right]^{T}=\sum_{\alpha} c_{\alpha}(\tau) \chi_{\alpha}(\mathbf{x})$, where the condition $\int d \mathbf{x} \chi_{\alpha}(\mathbf{x})^{\dagger} \chi_{\alpha^{\prime}}(\mathbf{x})=\delta_{\alpha, \alpha^{\prime}}$ is satisfied. In the adiabatic approximation,

$$
S_{e}\left[c^{*}, c\right]=\frac{1}{2} \int_{0}^{\hbar \beta} d \tau \sum_{\alpha} \epsilon_{\alpha} c_{\alpha}^{*}(\tau) c_{\alpha}(\tau) .
$$

The part of the action that describes the coupling becomes

$$
S_{c}=\frac{\hbar}{2} \int_{0}^{\hbar \beta} d \tau\left[\sum_{\alpha} \Gamma_{i \alpha}^{*} c_{\alpha}(\tau)-c_{\alpha}^{*}(\tau) \Gamma_{i \alpha}\right] \dot{r}_{v}^{i}(\tau),
$$

with $\Gamma_{i \alpha} \equiv \frac{1}{2} \int d \mathbf{x}\left[\chi_{\alpha}^{1 *}(\mathbf{x}) \partial_{i} \phi_{v}(\mathbf{x})-\chi_{\alpha}^{2 *}(\mathbf{x}) \partial_{i} \phi_{v}^{*}(\mathbf{x})\right]$. Note that the above expression is written in terms of $c_{\alpha}$ and $c_{\alpha}^{*}$ by using the relation that $\delta \phi^{*}(\mathbf{x}, \tau)$ is the complex conjugate of $\delta \phi(\mathbf{x}, \tau)$.

Since the fluctuation action is Gaussian, we can perform the integral over the environment fields $c_{\alpha}^{*}(\tau)$ and $c_{\alpha}(\tau)$ analytically. The effective action of the vortex is then given by

$$
S_{\mathrm{eff}}\left[\mathbf{r}_{v}\right]=\int_{0}^{\hbar \beta} d \tau\left[2 \pi i n_{c} \hbar \frac{1}{2} \epsilon^{i j} r_{v}^{i}(\tau) \dot{r}_{v}^{j}(\tau)+\frac{1}{2} m_{v} \dot{r}_{v}^{i} \dot{r}_{v}^{i}\right],
$$

with the effective mass of the vortex given by

$$
m_{v}=\hbar^{2} \sum_{\alpha}^{\prime} \frac{\left|\Gamma_{x \alpha}\right|^{2}}{\epsilon_{\alpha}}=\hbar^{2} \sum_{\alpha}^{\prime} \frac{\left|\Gamma_{y \alpha}\right|^{2}}{\epsilon_{\alpha}} .
$$

Note that since the units of $\Gamma_{i \alpha}$ are inverse length, $m_{v}$ indeed has the dimension of mass. The prime on the summation implies omission of the zero modes, as required by the 
constraint. The vortex equation of motion corresponding to this action contains the Magnus force

$$
\mathbf{F}_{M}=m_{v} \frac{d^{2} \mathbf{r}_{v}}{d t^{2}}=2 \hbar \pi n_{c}\left(\hat{z} \times \frac{d \mathbf{r}_{v}}{d t}\right),
$$

where the effective magnetic field strength is related to the Berry-phase coupling by the expression $B_{\text {eff }}=2 \hbar \operatorname{Im}\left[\Gamma_{x y}\right]$.

The vortex mass can be estimated by approximating the environment modes as vortices modulated by plane waves. This is a good approximation as long as we are interested in low-energy fluctuations in which vortex motion induces slowly varying nonuniformities. The phase- and density environmental-fluctuation modes in the presence of the vortex are then given, respectively, by

$$
\chi_{\mathbf{k}}^{\theta}=\frac{i}{\sqrt{2 n_{c} V}}\left(\begin{array}{c}
\phi_{v} \\
-\phi_{v}^{*}
\end{array}\right) e^{i \mathbf{k} \cdot \mathbf{x}}
$$

and

$$
\chi_{\mathbf{k}}^{\rho}=\frac{1}{\sqrt{2 n_{c} V}}\left(\begin{array}{c}
\phi_{v} \\
\phi_{v}^{*}
\end{array}\right) e^{i \mathbf{k} \cdot \mathbf{x}},
$$

where $V$ is the area of the system. Since these modes are spread over the full area of the superfluid, the corresponding eigenenergies can be approximated by their values in the absence of a vortex: $\epsilon_{\mathbf{k}}^{\theta}=\epsilon_{\mathbf{k}}=\hbar^{2} \mathbf{k}^{2} / 2 m$ and $\epsilon_{\mathbf{k}}^{\rho} \approx \epsilon_{\mathbf{k}}+2 g n_{c}$. When $\mathbf{k}=\mathbf{0}$, these reduce to global phase and density modes, respectively.

We now use these approximations to evaluate the coupling of a vortex to its environment. Separating the condensate wave function in the presence of a vortex into amplitude and phase factors $\left(\phi_{v}=\left|\phi_{v}\right| e^{i \theta}\right)$, we have that

$$
\partial_{x} \phi_{v}(\mathbf{x})=i\left|\phi_{v}\right| e^{i \theta} \partial_{x} \theta(\mathbf{x})+e^{i \theta} \partial_{x}\left|\phi_{v}(\mathbf{x})\right|,
$$

from which it follows that the coupling coefficients are given by

$$
\Gamma_{\mathbf{k} x}^{\theta}=\frac{-i}{\sqrt{2 n_{c} V}} \int d \mathbf{x} e^{-i \mathbf{k} \cdot \mathbf{x}}\left|\phi_{v}\right| \partial_{x}\left|\phi_{v}\right|
$$

and

$$
\Gamma_{\mathbf{k} x}^{\rho}=\frac{i}{\sqrt{2 n_{c} V}} \int d \mathbf{x} e^{-i \mathbf{k} \cdot \mathbf{x}}\left|\phi_{v}\right|^{2} \partial_{x} \theta .
$$

The vortex-core size provides the key length scale for vortexcondensate coupling. Since the core size corresponds to the coherence length scale $\xi$ at which kinetic and interaction energy scales balance $\left(\hbar^{2} / 2 m \xi^{2}=g n_{c}\right)$, coupling is expected to be large only when $k \xi$ is small, where $k=|\mathbf{k}| . \Gamma_{\mathbf{k} x}^{\theta}$ is induced by changes in wave-function amplitude which are nonzero only inside the vortex core. Choosing polar coordinates centered on the vortex position and approximating $\left|\phi_{v}\right|$ by $\sqrt{n_{c}}(r / \xi)$ inside the vortex core and by a constant $\sqrt{n_{c}}$ outside the core with $\partial_{x} r=-e^{i \mathbf{k} \cdot \mathbf{x}}\left(d / d k_{x} e^{-i \mathbf{k} \cdot \mathbf{x}}\right) / i r$, we obtain that

$$
\begin{aligned}
\Gamma_{\mathbf{k} x}^{\theta} & \simeq-\frac{1}{\sqrt{2 n_{c} V}} \frac{n_{c}}{\xi^{2}} \frac{d}{d k_{x}} \int_{\mathbf{x} \in \mathrm{core}} d \mathbf{x} e^{-i \mathbf{k} \cdot \mathbf{x}} \\
& \simeq \frac{k_{x}}{\sqrt{2 n_{c} V}} \frac{n_{c} \pi \xi^{2}}{4}
\end{aligned}
$$

for small $k \xi$. For the coupling to density fluctuations we note that the dominant contribution comes from outside the vortex cores and write $\partial_{x} \theta=-\sin (\theta) / r$ as $e^{i \mathbf{k} \cdot \mathbf{x}}\left(d / d k_{y} e^{-i \mathbf{k} \cdot \mathbf{x}}\right) / i r^{2}$ to obtain

$$
\begin{aligned}
\Gamma_{\mathbf{k} x}^{\rho} & \simeq \frac{2 \pi n_{c}}{\sqrt{2 n_{c} V}} \frac{d}{d k_{y}} \int_{k \xi}^{\infty} d x \frac{J_{0}(x)}{x} \\
& \simeq \frac{-2 \pi n_{c}}{\sqrt{2 n_{c} V}} \frac{k_{y}}{k^{2}}
\end{aligned}
$$

The final form for Eq. (25) is valid for small $k \xi$.

Since the coupling between the vortex and its environment must be dominated by modes with small $k \xi$, we can use these results to estimate the contributions of both phase $(\theta)$ and density $(\rho)$ fluctuations to the vortex mass. Substituting Eqs. (24) and (25) into Eq. (17) and using $2 \pi / \xi$ as an ultraviolet cutoff for the $m_{v}^{\theta}$ evaluation, we find that

$$
m_{v}=m_{v}^{\theta}+m_{v}^{\rho} \approx m\left(n_{c} \pi \xi^{2}\right)\left[\frac{\pi^{2}}{32}+\frac{\ln (R / \xi)}{2}\right] .
$$

The prefactor in this expression is the total mass of all boson particles inside the vortex core; the two factors inside square brackets are estimates of the $m_{v}^{\theta}$ and $m_{v}^{\rho}$ contributions. Viewing the spatial cutoff $R$ as the smaller of the system size and the distance between vortices, we see that the vortex mass will normally be dominated by the second term inside brackets, which represents coupling to density fluctuations, especially so when the fraction of the system area occupied by vortex cores is small. We comment later that the adiabatic approximation provides a good description of vortex quantum mechanics only when the logarithmic enhancement of the vortex mass due to the its $1 / r$ phase tail plays a substantial role. In general, both contributions to the vortex mass should be retained.

\section{Beyond the adiabatic limit}

We have shown that we can reproduce the commonly employed vortex-particle approximation by making an adiabatic approximation to our effective action. The next step is to scrutinize the adequacy of the adiabatic approximation. We expand the fluctuating vortex and environment fields,

$$
\begin{aligned}
& c_{\alpha}(\tau)=\frac{1}{\sqrt{\hbar \beta}} \sum_{n} c_{\alpha, n} e^{-i \omega_{n} \tau} \\
& c_{\alpha}^{*}(\tau)=\frac{1}{\sqrt{\hbar \beta}} \sum_{n} c_{\alpha, n}^{*} e^{i \omega_{n} \tau} \\
& r_{v}^{j}(\tau)=\frac{1}{\sqrt{\hbar \beta}} \sum_{n} r_{v, n}^{j} e^{-i \omega_{n} \tau},
\end{aligned}
$$

in Matsubara frequencies $\omega_{n}=\frac{2 \pi n}{\hbar \beta}$. The vortex action is derived by integrating over $c_{\alpha, n}$ and $c_{\alpha, n}^{*}$ as in the adiabatic case. The resulting action,

$$
S_{\mathrm{eff}}\left[\mathbf{r}_{v}\right]=\sum_{n} \pi \omega_{n} \hbar n_{c} \epsilon^{j k} r_{v, n}^{j} r_{v, n}^{k}+\frac{1}{2} \omega_{n}^{2} m_{v}^{j k}\left(i \omega_{n}\right) r_{v, n}^{j} r_{v, n}^{k},
$$

is of the same form as the adiabatic approximation action except that the mass becomes frequency dependent:

$$
m_{v}^{j k}\left(i \omega_{n}\right) \equiv \hbar^{2} \sum_{\alpha^{\prime}, \alpha} K_{\alpha^{\prime}, \alpha}\left(i \omega_{n}\right) \Gamma_{r_{v}^{j}, \alpha^{\prime}} \Gamma_{r_{v}^{k}, \alpha}^{*},
$$


where

$$
K_{\alpha, \alpha^{\prime}}\left(i \omega_{n}\right)=\left[\epsilon_{\alpha} \delta_{\alpha^{\prime}, \alpha}-2 i \hbar \omega_{n} \Gamma_{\alpha^{\prime}, \alpha}\right]^{-1} .
$$

The mass kernel captures both the renormalization of the effective mass at larger frequencies and the dissipative coupling of the vortex to the condensate environment. The quantity

$$
\Gamma_{\alpha^{\prime}, \alpha}=\frac{1}{2} \int d V\left[\chi_{\alpha^{\prime}}^{1 *}(\mathbf{x}) \chi_{\alpha}^{1}(\mathbf{x})-\chi_{\alpha^{\prime}}^{2 *}(\mathbf{x}) \chi_{\alpha}^{2}(\mathbf{x})\right]
$$

is the Berry-phase coupling between environment fluctuations which we have ignored in the adiabatic theory.

Approximating, as before, density and phase fluctuations by plane waves, we replace state labels $\alpha^{\prime}, \alpha$ by momentum labels $\mathbf{k}^{\prime}, \mathbf{k}$. We divide the Berry-phase coupling between fluctuations $\Gamma_{\mathbf{k}^{\prime}, \mathbf{k}}$ into different categories. Using Eqs. (19) and (20) we find that

$$
\Gamma_{\mathbf{k}^{\prime}, \mathbf{k}}^{\rho \theta}=\frac{i}{2} \delta_{\mathbf{k}^{\prime}, \mathbf{k}} \quad \Gamma_{\mathbf{k}^{\prime}, \mathbf{k}}^{\theta \rho}=-\frac{i}{2} \delta_{\mathbf{k}^{\prime}, \mathbf{k}}
$$

and that there is no coupling among phase fluctuations and density fluctuations. In this approximation then, the matrix $K_{\alpha^{\prime}, \alpha}\left(i \omega_{n}\right)$ is given by $K_{\alpha^{\prime}, \alpha}=K_{\mathbf{k}}\left(i \omega_{n}\right) \delta_{\mathbf{k}^{\prime}, \mathbf{k}}$, with the matrix $K_{\mathbf{k}}$ given by

$$
\begin{aligned}
K_{\mathbf{k}}\left(i \omega_{n}\right) & =\left(\begin{array}{cc}
\epsilon_{\mathbf{k}}^{\theta} & -\hbar \omega_{n} \\
\hbar \omega_{n} & \epsilon_{\mathbf{k}}^{\rho}
\end{array}\right)^{-1} \\
& =\frac{1}{\left(\epsilon_{\mathbf{k}}^{\theta} \epsilon_{\mathbf{k}}^{\rho}+\hbar^{2} \omega_{n}^{2}\right)}\left(\begin{array}{cc}
\epsilon_{\mathbf{k}}^{\rho} & \hbar \omega_{n} \\
-\hbar \omega_{n} & \epsilon_{\mathbf{k}}^{\theta}
\end{array}\right) .
\end{aligned}
$$

To study damping, we perform a Wick rotation $i \omega_{n} \rightarrow \omega+$ $i 0 \equiv \omega^{+}$. The poles of the matrix elements $K_{\mathbf{k}}\left[\omega^{+}\right]$are the Bogoliubov modes with excitation energy $E_{\mathbf{k}}^{b}=\left[\frac{\hbar^{2} k^{2}}{2 m}\left(\frac{\hbar^{2} k^{2}}{2 m}+\right.\right.$ $\left.\left.2 g n_{c}\right)\right]^{1 / 2}$.

We now derive approximate analytic results for the nonadiabatic vortex action. The key step is to sum over environment-fluctuation modes to obtain an expression for the frequency-dependent mass kernel $m_{v}^{j j}$, where $j \in x, y$ :

$$
\begin{aligned}
m_{v}^{j j}\left(i \omega_{n}\right)= & 2 \hbar^{2} \sum_{\mathbf{k}}\left(\Gamma_{x \mathbf{k}}^{\theta *} \Gamma_{x \mathbf{k}}^{\rho *}\right) K_{\mathbf{k}}\left(i \omega_{n}\right)\left(\begin{array}{c}
\Gamma_{x \mathbf{k}}^{\theta} \\
\Gamma_{x \mathbf{k}}^{\rho}
\end{array}\right) \\
= & 2 \hbar^{2} \sum_{\mathbf{k}} \frac{1}{E_{b}^{2}(k)-\left(i \hbar \omega_{n}\right)^{2}}\left[2 g n_{c}\left|\Gamma_{x \mathbf{k}}^{\theta}\right|^{2}\right. \\
& \left.+\frac{\hbar^{2} k^{2}}{2 m}\left|\Gamma_{x \mathbf{k}}^{\rho}\right|^{2}+\hbar \omega_{n}\left(\Gamma_{x \mathbf{k}}^{\rho *} \Gamma_{x \mathbf{k}}^{\theta}-\Gamma_{x \mathbf{k}}^{\rho} \Gamma_{x \mathbf{k}}^{\theta *}\right)\right]
\end{aligned}
$$

At small $\mathbf{k}$ the Bogoliubov excitation energy takes its phonon dispersion limit, $E_{b}=\hbar k\left(g n_{c} / m\right)^{1 / 2}=\hbar k c$, where $c$ is the speed of sound in the superfluid. Note that the final term in square brackets vanishes.

We proceed as in the adiabatic limit, separating the mass into phase $m_{\theta}$ and density $m_{\rho}$ fluctuation contributions:

$$
\begin{aligned}
m_{\theta}= & \hbar^{2} \sum_{\mathbf{k}}\left[\frac{1}{E_{b}(k)-\hbar \omega-i \delta}+\frac{1}{E_{b}(k)+\hbar \omega+i \delta}\right] \\
& \times \frac{2 g n_{c}\left|\Gamma_{x \mathbf{k}}^{\theta}\right|^{2}}{E_{b}(k)}
\end{aligned}
$$

$$
\begin{aligned}
& \simeq \frac{\pi g n_{c}^{2} \xi^{4}}{32 c^{4}} \int_{2 \pi c / R}^{2 \pi c / \xi} d \omega^{\prime}\left[\frac{\omega^{\prime 2}}{\omega^{\prime}+\omega}+P\left[\frac{\omega^{\prime 2}}{\omega^{\prime}-\omega}\right]\right. \\
& \left.+i \pi \omega^{2} \delta\left(\omega^{\prime}-\omega\right)\right] .
\end{aligned}
$$

Similarly, the condensate-density-fluctuation contribution $m_{\rho}$ is

$$
\begin{aligned}
m_{\rho}= & 2 \hbar^{2} \sum_{\mathbf{k}} \frac{1}{E_{b}(k)}\left[\frac{1}{E_{b}(k)-\hbar \omega-i \delta}\right. \\
& \left.+\frac{1}{E_{b}(k)+\hbar \omega+i \delta}\right] \frac{\hbar^{2} k^{2}}{4 m}\left|\Gamma_{x \mathbf{k}}^{\rho}\right|^{2} \\
\simeq & \frac{m \pi \xi^{2} n_{c}}{4} \int_{\omega}^{2 \pi c / \xi} d \omega^{\prime}\left[\frac{1}{\omega^{\prime}+\omega}+P\left(\frac{1}{\omega^{\prime}-\omega}\right)\right. \\
& \left.+i \pi \delta\left(\omega^{\prime}-\omega\right)\right] \simeq \frac{m \pi \xi^{2} n_{c}}{4}[2 \ln (2 \pi c / \omega \xi)+i \pi] .
\end{aligned}
$$

Assuming that the density coupling is dominant, we see that the dissipative contribution to the mass is frequency independent and that the real part of the mass has a corresponding logarithmic dependence on frequency. This result is similar to that of Arovas and Freire [30], obtained by different methods. The adiabatic limit result for the mass quoted previously corresponds to setting the frequency argument in this expression to its minimum value $\sim c / R$. The quality factor for vortex-cyclotron motion in the adiabatic limit is therefore

$$
\left(\omega_{c} \tau\right)_{\text {vortex }} \sim \ln (R / \xi) .
$$

Sharp cyclotron modes are expected only for very dilute vortices or for isolated vortices in a large condensed cloud.

We can now comment on the validity of using the adiabatic approximation to describe vortex quantum fluctuations. As a criterion for the validity of the adiabatic theory in the continuum limit, we require that the adiabatic cyclotron frequency $\omega_{c}$ be much smaller than $c / \xi$, that is,

$$
\omega_{c}=8 \frac{\hbar}{m \xi^{2} \ln \frac{R}{\xi}} \ll \frac{c}{\xi} \simeq \frac{\hbar}{m \xi^{2}} .
$$

This inequality is satisfied only if $R$ or the mean distance between vortices is at least a thousand times $\xi$, that is, only if an extremely small fraction of the system area is occupied by vortex cores. This condition seems to be quite difficult to satisfy for a cold-atom bosonic system. In the next section we explore the possibility of altering this comparison using the condensate tuning possibilities available in optical lattices.

\section{VORTEX CYCLOTRON MODES IN OPTICAL LATTICES}

\section{A. Boson Hubbard model}

Cold atoms in optical lattices offer the opportunity to alter vortex physics by tuning system parameters like the interaction strength between atoms and the optical potential depth and period. When an optical-lattice potential is sufficiently deep, low-energy physics is described by a one-band Hubbard model $[7,8]$. This standard boson Hubbard model without an external 
trapping potential is described by the Hamiltonian

$$
H_{\mathrm{BH}}=-J \sum_{\langle i, j\rangle} b_{i}^{\dagger} b_{j}+\frac{U}{2} \sum_{j} b_{j}^{\dagger} b_{j}^{\dagger} b_{j} b_{j}-\mu \sum_{j} b_{j}^{\dagger} b_{j} .
$$

Here $\langle i, j\rangle$ represents summation over the nearest neighbors, $J$ is the tunneling amplitude, $U$ is the interaction energy between atoms on the same site, and $\mu$ is the chemical potential. The energy functional $\mathcal{E}$ in the coherent state path integral action for the boson Hubbard model is correspondingly given by

$$
E_{\mathrm{SF}}=-J \sum_{<i, j>} \phi_{i}^{*} \phi_{j}+U \sum_{j} \phi_{j}^{*} \phi_{j}^{*} \phi_{j} \phi_{j}-\mu \sum_{j} \phi_{j}^{*} \phi_{j} .
$$

Mean-field configurations of the condensate are extrema of this energy functional and therefore satisfy the corresponding Gross-Pitaevskii (GP) equation. The boson Hubbard model on a $2 \mathrm{D}$ square lattice with lattice constant $D$ can be viewed as a discretization of the $2 \mathrm{D}$ continuum model we have been discussing heretofore with the correspondences

$$
J \leftrightarrow \frac{\hbar^{2}}{2 m D^{2}}
$$

and

$$
U \leftrightarrow \frac{g}{D^{2}} .
$$

Based on continuum-model considerations explained earlier, we expect the adiabatic approximation to be satisfied when the ratio of the number of lattice sites per vortex to the number of lattice sites occupied by a vortex core is large. In searches for vortex-Landau quantization then, the small vortex cores produced by strong interactions are favored. On the other hand, we know that the Bose condensate is destroyed by quantum fluctuations for [34] $U>U_{c} \simeq 4 J z \bar{n}$, where $z$ is the lattice coordination number and $\bar{n}$ is the average number of bosons per lattice site ( $z=4$ in our case). Using the continuum-model correspondences, we estimate that

$$
\frac{\xi^{2}}{D^{2}} \sim \frac{J}{U} \sim \frac{U_{c}}{U} \frac{1}{4 z \bar{n}} .
$$

There should therefore be a broad range of interaction strengths over which superfluidity survives and vortex cores are concentrated in a single square-lattice plaquette, even for the small values of $\bar{n}$ typical in optical-lattice systems. The numerical calculations reported on later in this article explore the possibility, suggested by the approximate continuummodel calculations, that sharp adiabatic-limit vortex cyclotron motion modes might occur for strong $U$, but still smaller than $U_{c}$.

\section{B. Vortex displacement fluctuations}

In this section we describe numerical calculations based on the theoretical formulation outlined in Sec. II, which demonstrate the possibility of achieving some degree of experimental control over the sharpness of the vortex cyclotron resonance signal. To find solutions of the square-lattice Hubbard model GP equations which contain vortices, we impose quasiperiodic boundary conditions on a finite lattice with size $L \times L$. We first impose a phase winding of $2 \pi$ on this area by using the boundary conditions

$$
\begin{gathered}
\psi\left(x, \frac{L}{2}\right)=e^{i \pi\left(1-\frac{x}{L}\right)} \psi\left(x,-\frac{L}{2}\right) \\
\psi\left(\frac{L}{2}, y\right)=e^{-i \pi\left(1-\frac{x}{L}\right)} \psi\left(-\frac{L}{2}, y\right),
\end{gathered}
$$

where the origin is chosen at the center of the computational grid, which is also the center of the vortex core. We then minimize the energy by integrating the time-dependent GP equations over imaginary time,

$$
\psi_{i, j}^{n+1}-\psi_{i, j}^{n}=-\epsilon H_{\mathrm{GP}} \psi_{i, j}^{n},
$$

carefully restoring the normalization $\int d^{2} \mathbf{x}\left|\psi_{i, j}^{n+1}\right|^{2}=N_{a}$ after each time step and starting from an ansatz in which the phase winds at a constant rate around the boundary. Here $i, j$ are labels for the two-dimensional lattice points, the label $n$ counts steps in an imaginary time evolution with time step $\epsilon$, and $N_{a}=$ $\bar{n} L^{2} / D^{2}$ is the number of atoms in the $L \times L$ computational cell.

The energy functional for fluctuations around the vortex ground state are described by the lattice version of the hermitian Hamiltonian in Eq. (11). Diagonalizing this Hamiltonian, we always find a zero-energy global-phase mode and two other low but finite energy-fluctuation modes which we identify as vortex-translational modes. The vortex modes do not have precisely zero energy in our simulations because of lattice pinning and because of the boundary conditions we apply to the simulation cell. The number of eigenvalues of $\mathcal{H}$ is twice the number of atoms in the simulation cell. In order to connect approximately with an infinite system with a finite density of vortices, we broaden this discrete spectra into continua in the spectral calculations described below by replacing $\delta$-functions at eigenenergies by Lorentzian functions with a width $\delta \sim \hbar^{2} / 2 m L^{2}$.

We report results for four different interaction strengths, $U=0.05 \mathrm{~J}, U=0.40 \mathrm{~J}, U=2.25 \mathrm{~J}$, and $U=3.80 \mathrm{~J}$, which we refer to later in this article as very weak, weak, strong, and very strong, respectively. According to our estimates, only the very weak and weak cases should yield vortex cores which extend over many square-lattice plaquettes and this expectation is confirmed in Fig. 1. All our calculations were performed for a square-lattice system with one vortex per $N_{\text {site }}=46 \times 46=2116$ lattice sites. In order to compare with potential future optical-lattice experiments, we choose the number of atoms per site $\bar{n}=1$ in all the calculations described below. Our approach, which is designed to describe systems with weak and moderately strong interactions, is complementary to that taken by Lindner et al. [35], who use many-body exact diagonalization methods to estimate vortex properties and concentrate on the case of a half-filled lattice with infinitely strong on-site repulsive interactions.

The two degenerate vortex-translation modes are identified as $c_{x}(\tau) \delta \phi_{0 x}$ and $c_{y}(\tau) \delta \phi_{0 y}$, corresponding to vortex displacements along the $x$-axis and $y$-axis of the lattice, respectively. Here $\delta \phi_{0 i}$ is a normalized eigenfunction of the system energy functional and is obtained numerically. To make contact with the vortex coordinate $\mathbf{r}_{v}=\left[x_{v}(\tau), y_{v}(\tau)\right]$ in our continuum field theory, we can associate the excitation amplitudes $c_{x}, c_{y}$ 
(a)

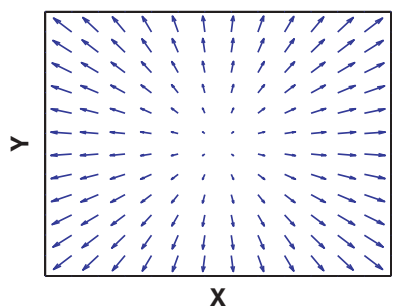

(c)

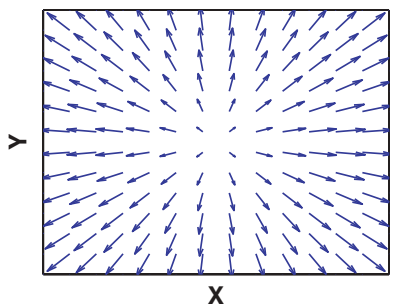

(b)

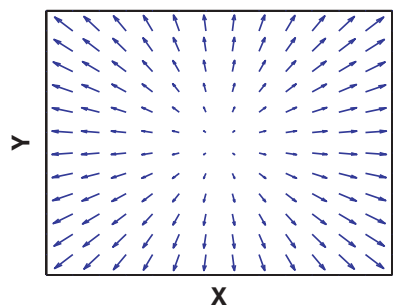

(d)

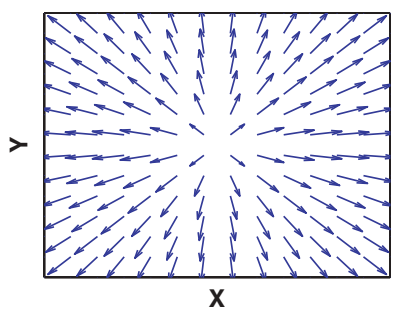

FIG. 1. (Color online) Condensate amplitude and phase for the vortex-state configuration indicated by the length and orientation of an arrow on a set of $12 \times 12$ sites near the center of the $46 \times 46$ site simulation cell: (a) $U / J=0.05$, (b) $U / J=0.40$, (c) $U / J=2.25$, and (d) $U / J=3.8$. The continuum-model estimate for the vortex-core size in square-lattice constant units is $\xi=$ $D(J / U)^{1 / 2}=4.4721 D, 1.5811 D, 0.6667 D, 0.5130 D$ for (a), (b), (c), and (d) respectively.

with the vortex coordinates $x_{v}, y_{v}$ using the relations

$$
\begin{aligned}
& c_{x} \delta \phi_{0 x}=-x_{v} \partial_{x} \phi_{v} \\
& c_{y} \delta \phi_{0 y}=-y_{v} \partial_{y} \phi_{v} .
\end{aligned}
$$

This identification becomes exact in the continuum limit where $\phi_{v}\left[\mathbf{x}-\mathbf{r}_{v}(\tau)\right]=\phi_{v}(\mathbf{x})-\mathbf{r}_{\mathbf{v}} \cdot \nabla \phi_{\mathbf{v}}$ for small vortex displacements. In this limit $c_{x}$ and $c_{y}$ represent precisely the same degrees of freedom as $x_{v}$ and $y_{v}$ and differ only by a multiplicative constant:

$$
\frac{x_{v}}{c_{x}}=\frac{y_{v}}{c_{y}}=-\left[\frac{1}{\int d \mathbf{x}\left|\partial_{x} \phi_{v}\right|^{2}}\right]^{1 / 2} .
$$

In the continuum limit,

$$
\begin{aligned}
\frac{x_{v}}{c_{x}} & =\int d \mathbf{x}\left[f^{2}\left(\partial_{x} \theta\right)^{2}+\left(\partial_{x} f\right)^{2}\right]^{-1 / 2} \\
& \approx\left[\pi n_{c}(1+\ln R / \xi)\right]^{-1 / 2} .
\end{aligned}
$$

When we turn to the Berry-phase coupling coefficients for the lattice-model fluctuations later in this article, they are first evaluated using Eq. (31) with the numerical eigenstates of the energy functional. Applying a central difference approximation, $\partial_{x} \phi_{v}(i, j) \simeq\left[\phi_{v}(i+1, j)-\phi_{v}(i-1, j)\right] / 2 D$, to the vortex solutions of our lattice model $(i, j$ are the lattice point indices), Eq. (47) then allows us to appropriately renormalize those coupling coefficients that involve vortex-displacement coordinates. This identification of particular fluctuation modes as vortex displacements is necessary in order to evaluate the adiabatic vortex mass using Eq. (17), or its frequencydependent nonadiabatic generalization using Eq. (29), but drops out of any direct calculation of a physical observable.

(a)

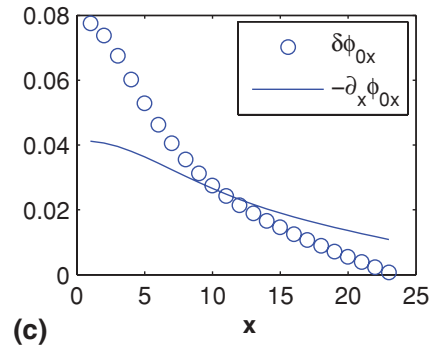

(b)
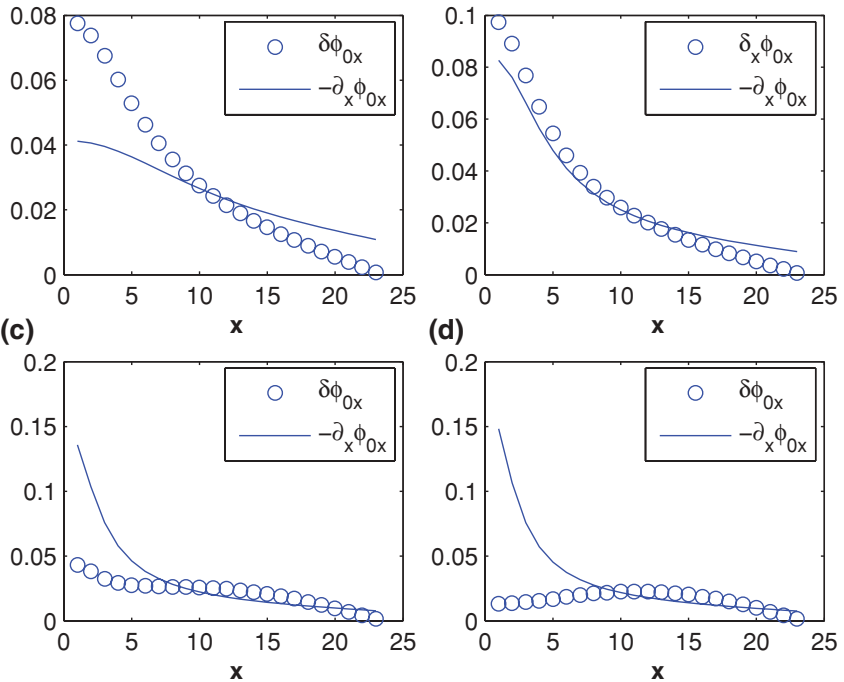

(d)

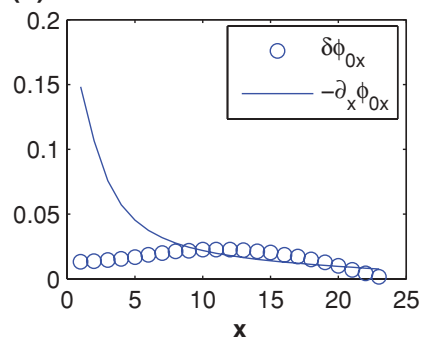

FIG. 2. (Color online) Comparison of the lattice-model energy functional eigenstate identified as a vortex displacement in the $\hat{x}$ direction $\left[\delta \phi_{0 x}(\mathbf{x})\right.$, circles] with the renormalized finite-difference derivative of the lattice-model vortex wave functions (solid line) illustrated in Fig. 1 for the same four values of $U / J$. Distance in this figure is in units of the lattice constant $D$ of the optical lattice.

In particular, the renormalization does not influence the frequency or broadening of the mode which we identify as a vortex cyclotron resonance. Its only role is related to the physical identification of a particular low-frequency collective fluctuation coordinate as the position of a well-defined texture of the condensate field. The identification becomes less well founded, of course, as the vortex cores become smaller and their locations more strongly pinned by the lattice. We will return to this point frequently later in this article.

In Fig. 2, we compare the normalized vortex-displacement mode $\delta \phi_{0 x}(\mathbf{x})$ obtained by diagonalizing the energy functional in Eq. (11) with the renormalized finite-difference approximation to the derivative, $-\partial_{x} \phi_{v}$, of the vortex condensate configurations illustrated in Fig. 1. For the weak-interaction cases [Figs. 2(a) and 2(b)], the close comparison between the two functions clearly justifies the identification. In Fig. 2(a), the vortex-core size is not very much smaller than our simulation cell size and we attribute differences between the two functions to boundary effects. In Fig. 2(b), the vortex core is smaller and boundary effects are less important. In the strong-interaction cases [Figs. 2(c) and 2(d)], we see the influence of lattice pinning which suppresses the numerical fluctuation mode close to the vortex core, more so for stronger interactions. The ratio between $\delta \phi_{0 x}(\mathbf{x})$ and $-\partial_{x} \phi_{v}$ implied by the analytical estimate [Eq. (48)] varies from $\sim 0.31 D$ in the weak-interaction limit to $\sim 0.24 D$ for the strong-interaction limit and is in good qualitative agreement the scaling factors used to normalize $-\partial_{x} \phi_{0 x}$ in Fig. 2 .

\section{Vortex effective mass}

As explained earlier, the vortex effective mass is due to coupling between the vortex displacements and their 
(a)

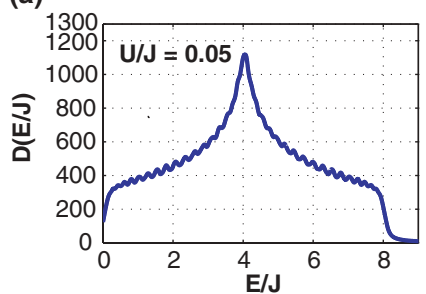

(c)

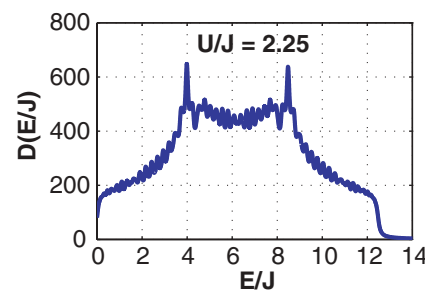

(b)

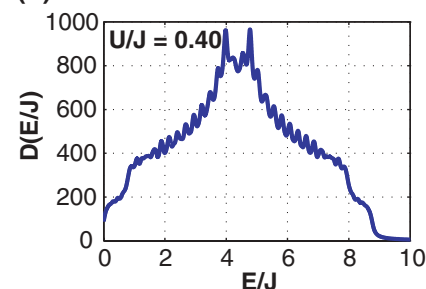

(d)

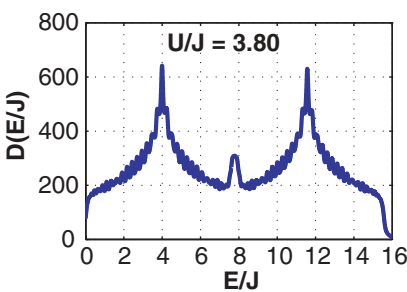

FIG. 3. (Color online) Density of states $D\left(E_{R}\right)$ of environmentfluctuation modes as a function energy $E / J$ for $U / J=0.05$ (a), $U / J=0.40$ (b), $U / J=2.25$ (c), and $U / J=3.80$ (d).

environment which, in the Gaussian approximation we employ here, consists of condensate phase and density fluctuations. In Fig. 3, we plot the density-of-states for condensate-density and phase-fluctuation modes, distorted by the presence of the vortex. In the absence of a vortex we would expect that the phase modes should have energy $\epsilon_{\mathbf{k}}^{\theta}=2 J[2-$ $\cos \left(k_{x} a\right)-\cos \left(k_{y} a\right)$ ], varying over an $8 J$ range, and that the density-fluctuation modes have energy $\epsilon_{\mathbf{k}}^{\rho}=\epsilon_{\mathbf{k}}^{\theta}+2 U$. We see in Fig. 3 that the presence of the vortex does not have a large influence on the overall spectrum of fluctuations. The prominent features at $E=4 J$ and $E=4 J+2 U$ in Fig. 3 originate from the van-Hove singularities of the nearest-neighbor hopping model on the square lattice. As the interactions strengthen, the phase- and density-fluctuation spectra separate. Note that the densities of states differ by a factor of two between Fig. 3(a), in which the phase and density spectra overlap, and Fig. 3(d), in which they are nearly completely separated.

In Fig. 4 we plot a spectral decomposition of adiabaticvortex-mass contributions $d m_{v} / d E$ defined by

$$
m_{v}=\hbar^{2} \sum_{\alpha} \frac{\left|\Gamma_{\alpha x}\right|^{2}}{\epsilon_{\alpha}} \equiv \int d E \frac{d m_{v}}{d E} .
$$

In the very weak-interaction case [Fig. 4(a)], density- and phase-fluctuation contributions are not spectrally resolved. We see here that the mass is due mainly to low-energy long-wavelength-fluctuation modes. For Figs. 4(b)-4(d) we see separate contributions near $E=0$ due to phase fluctuations and near $E=2 U$ due to density fluctuations. As the interaction strengthens and the vortex-core radius gets smaller, the density-fluctuation contribution becomes relatively more important, in agreement with our continuum-model analytic results. These results emphasize the fact that for moderate vortex densities, both phase- and density-fluctuation contributions need to be included in calculating the vortex mass.

When we go beyond the adiabatic approximation, the kinetic coupling between environment phase and density fluctuations is retained. This coupling leads (in the Gaussian (a)

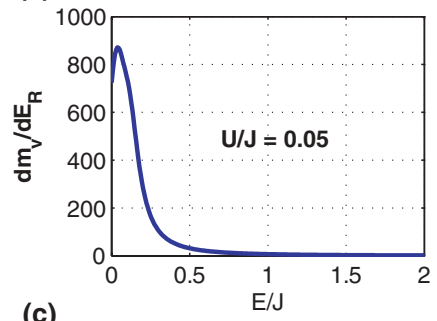

(c)

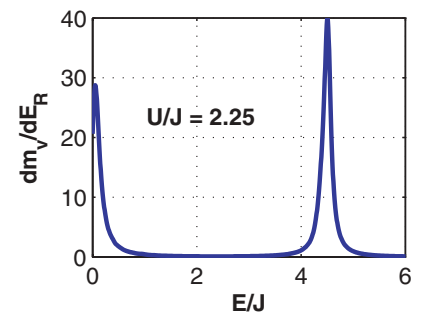

(b)

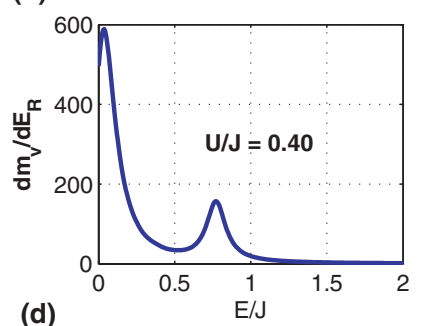

(d)

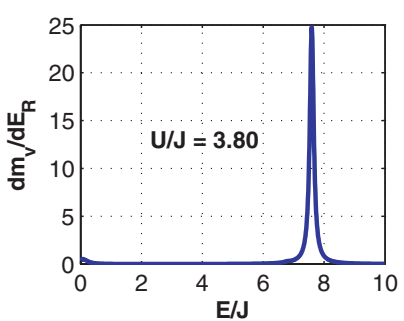

FIG. 4. (Color online) Spectral representation of adiabaticvortex-mass contributions $d m_{v} / d E$ vs mode energy $E / J$ for $U / J=$ 0.05 (a), $U / J=0.40$ (b), $U / J=2.25$ (c), and $U / J=3.80$ (d). Where a separation exists, the lower-energy contribution can be associated with coupling to phase fluctuations and the higher energy contribution with coupling to density fluctuations. The density-fluctuation contribution has a larger relative importance when interactions are strong.

fluctuation approximation) to Bogoliubov elementary excitations of the environment. In the absence of a vortex, the environment fluctuations can be labeled by wave vector $\mathbf{k}$ and the elementary-excitation energy is given by $E_{B}(\mathbf{k})=\left(\epsilon_{\mathbf{k}}^{\theta} \epsilon_{\mathbf{k}}^{\rho}\right)^{1 / 2}$, with characteristic linear dispersion at long wavelength. For each $\mathbf{k}$, the elementary-excitation energy is therefore intermediate between the energies of the corresponding phase and density modes. There is now a single branch of modes, instead of two different ones. As shown in the approximate continuummodel calculations, the phase and density contributions appear separately; their correlation functions have different residues at elementary-excitation poles. The mass becomes $\omega$ dependent and develops an imaginary part which captures the dissipative effect of environmental coupling. The sign of the contribution of a particular elementary excitation to the mass changes when $\omega$ becomes larger than its energy.

In Fig. 5 we plot the density of states $D_{B}(E)$ of Bogoliubov excitations in the presence of a vortex for the usual set of interaction strengths. It is clear that distortion by the vortex has little influence on such global indicators of environment properties. For weak interactions the density of states is roughly constant, except at very low energies, corresponding to quadratic dispersion at small $\mathbf{k}$, whereas for stronger interactions the density of states has a linear dependence of energy, corresponding to linearly dispersing phononlike elementary excitations.

Having characterized the environment modes, we now discuss the adiabatic vortex mass $m_{v}$ and Berry phase $\Gamma_{x y}$ for optical-lattice condensates. As explained earlier, both of these quantities are sensitive to the conversion factor between the vortex coordinate $x_{v}$ and the excitation amplitude of the corresponding normalized energy-fluctuation mode. The procedure we use to define this conversion factor, explained earlier in 
(a)
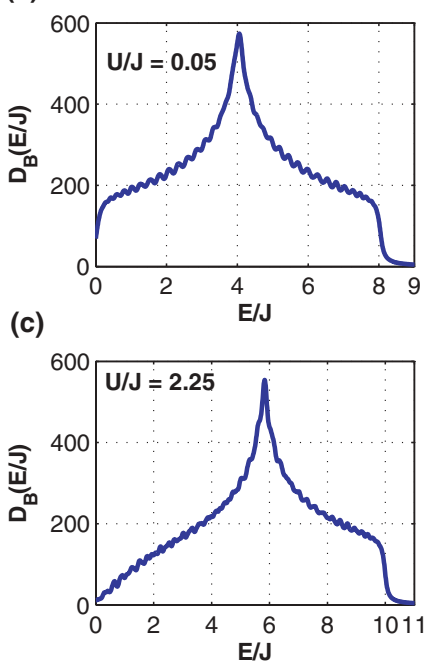

(b)

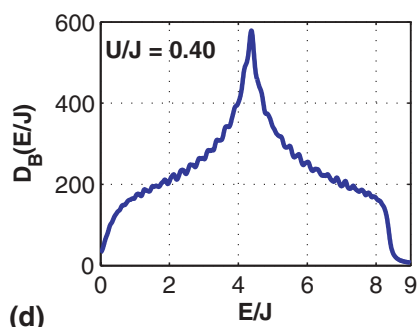

(d)

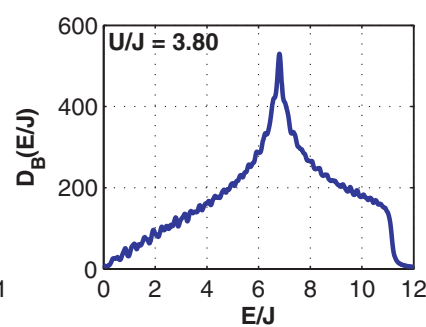

FIG. 5. (Color online) Spectral density of states of Bogoliubov excitations $D_{B}(E)$ as a function of $E / J$ for interaction strengths $U / J=0.05$ (a), $U / J=0.40$ (b), $U / J=2.25$ (c), and $U / J=$ 3.80 (d). The Bogoliubov modes have linear dispersion at long wavelengths and a corresponding linear density of states at low energies. At higher energies their density of states shows the characteristic van-Hove singularities of square-lattice systems with near-neighbor hopping. Linear dispersion holds over a wider energy range when the interactions are stronger.

this article, becomes somewhat arbitrary when interactions are very strong and vortices are strongly pinned by the lattice. The vortex cyclotron frequency, $\omega_{c}=2 \hbar\left|\Gamma_{x y}\right| / m_{v}$, is, however, independent of this conversion factor. This expression for $\omega_{c}$ follows from the effective action in Eq. (28) after Wick rotation to real frequencies using an adiabatic-approximation frequency-independent vortex mass.

In Fig. 6, we illustrate the dependence of the adiabatic vortex mass on the interaction strength $U / J$. When interactions are strong and vortex cores are small $(U / J \gtrsim 1)$, we see that the vortex mass is not much larger than the bare particle mass. In this strong-interaction regime our numerical adiabatic vortex masses agree reasonably well with the continuum-model expression translated into lattice-model parameters:

$$
\frac{m_{v}}{m} \simeq \frac{\pi \bar{n} J}{U}\left[\frac{\pi^{2}}{32}+\frac{\ln N}{4}+\frac{\ln (U / J)}{4}\right],
$$

where $N=2116$ is the number of lattice sites in our simulation cell. This expression is also plotted in Fig. 6. There is a reasonable degree of agreement between approximate analytic and numerical masses in the strong-interaction limit, even when the vortex-core size becomes comparable to $D$, possibly because mass contributions then come mainly from interactions with density fluctuations outside the vortex core. At weaker interactions, where the vortex cores become large, the deviations from the analytic expression are larger. In particular for very weak interactions, the analytic expression has $m_{v}$ increasing as $U^{-1}$, a trend that is not reproduced by our numerical calculations. We attribute this discrepancy to a violation in the numerical calculations of the assumption

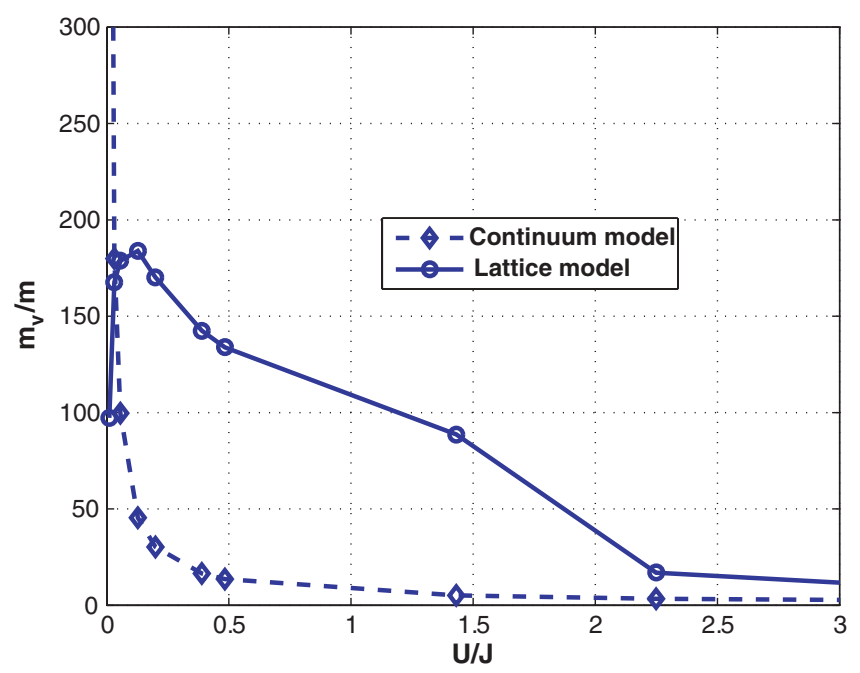

FIG. 6. (Color online) Vortex mass $m_{v}$ as a function of interaction strength $U / J$. The dashed line (diamonds) is a plot of the the analytical continuum-model expression [Eq. (26)] using the continuum-limit expression for the vortex-core size $\xi$. The solid line (circles) shows vortex masses evaluated numerically from Eq. (49). The numerical calculation is dependent on the conversion factor between the excitation amplitudes $c_{x}(\tau)$ and $x_{v}(\tau)$, as discussed in the text.

made in the analytic calculations that the vortex core size is small compared to $R$. At intermediate interaction strengths there is reasonable quantitative agreement between the analytic approximation, which is relatively crude when applied to a lattice model, and the numerical adiabatic mass results.

Next we discuss numerical results for $\Gamma_{x y}$, the Berry-phase coupling between $x_{v}$ and $y_{v}$. This coupling is the source of the effective magnetic field $B_{\text {eff }}$ responsible for vortex cyclotron resonance. In the continuum-model, $\Gamma_{x y}=\pi n_{c} \rightarrow$ $\pi \bar{n} / D^{2} \rightarrow \pi / D^{2}$. We see in Fig. 7 that the numerical latticemodel $\Gamma_{x y}$ is in agreement only at intermediate values of $U / J$. We attribute deviations at small $U / J$ to the finite size of our simulation cell, as in the adiabatic effective mass discussion. However, $\Gamma_{x y}$ also deviates strongly from the analytic result at large values of $U / J$. We attribute this to the fact that vortex cores in this regime are localized mainly in the central plaquette of the lattice. As a result, the condensate-density variation is almost negligible across the entire lattice. Since the continuum-model $\Gamma_{x y}$ is proportional to the difference between the condensate density far away from and at the center of the vortex core, the small values obtained for $\Gamma_{x y}$ in this limit are not surprising. The strong discrepancy compared to the continuum model in this regime is also due to lattice pinning effects which work against the identification of the low-energy-fluctuation modes with vortex translations.

Before turning to the full nonadiabatic calculations, we first discuss the adiabatic approximation for the vortex cyclotron frequency in the lattice model which is proportional to $\Gamma_{x y} / m_{v}$. Using Eqs. (26), (41), and (42), the continuum-model adiabatic cyclotron mode energy translates into the following latticemodel expression:

$$
E_{c} \simeq \frac{8 \bar{n} U}{\frac{\pi^{2}}{16}+\frac{\ln N}{2}+\frac{\ln (U / J)}{2}} .
$$




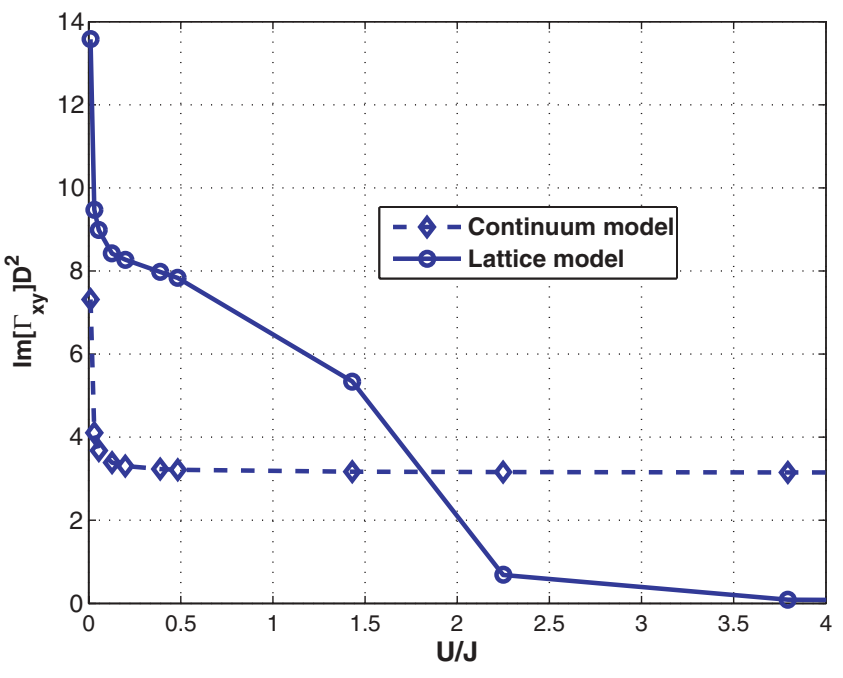

FIG. 7. (Color online) Berry-phase coupling $\operatorname{Im}\left[\Gamma_{x y}\right]=\left|\Gamma_{x y}\right|$ between $x_{v}$ and $y_{v}$ as a function of interaction strength $U / J$. The dashed line (diamonds) is the data calculated from Eq. (8) for the continuum model. The upturn at small $U / J$ reflects the fact that the condensate density evaluated at the simulation cell boundaries is increased compared to the average condensate density when the vortex cores occupy a significant fraction of the simulation cell area. The solid line (circles) is the data for the lattice model directly calculated from the wave function of the vortex-displaced modes $\delta \phi_{0 x}, \delta \phi_{0 y}$.

Based this expression, we should expect that the cyclotron mode frequency $E_{c} / J$ would scale with the interaction strength $\bar{n} U / J \rightarrow U / J$, as indicated by the dashed line in Fig. 8. The discrepancies between continuum-model and lattice-model results illustrated in Fig. 8 are mainly due to discrepancy in $\Gamma_{x y}$ values at large $U$ and due to large vortex-core sizes and related discrepancies in the vortex mass at small $U$. At intermediate values of $U$ the numerical lattice-

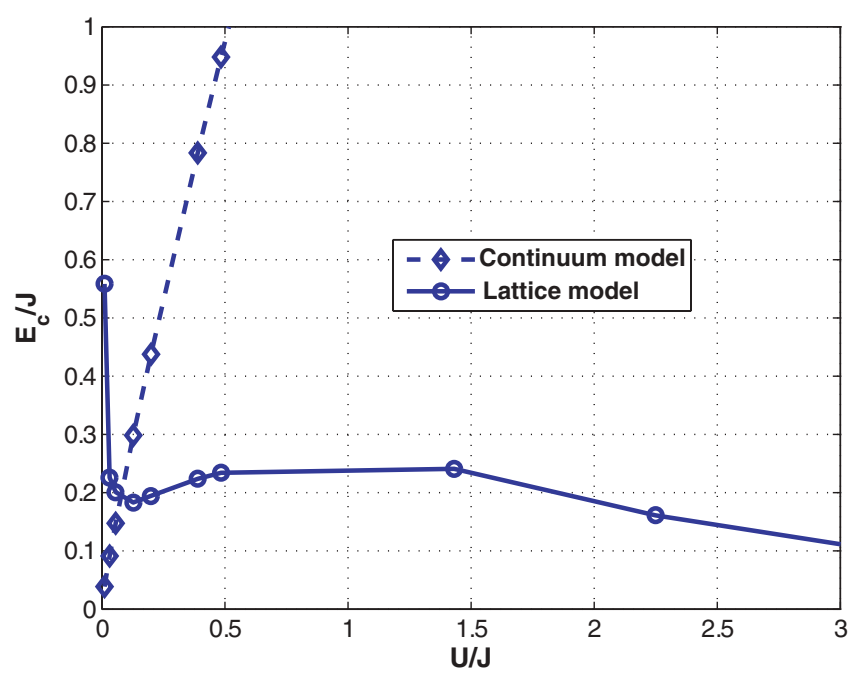

FIG. 8. (Color online) Vortex cyclotron frequency $E_{c} / J$ as a function of interaction $U / J$. The dashed line shows the continuummodel estimates while the solid line illustrates numerical latticemodel results. (a)

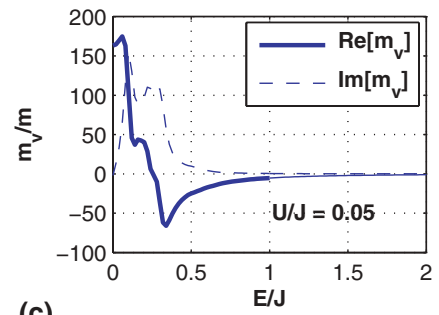

(c)

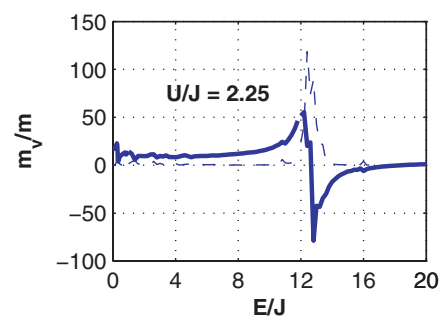

(b)

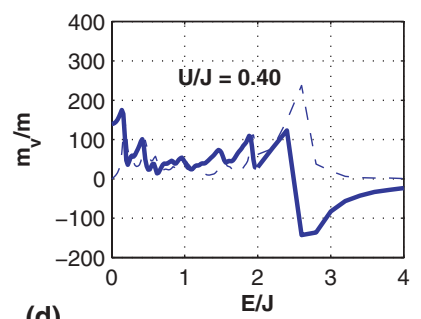

(d)

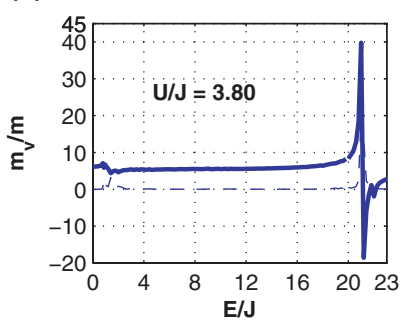

FIG. 9. (Color online) Real part and imaginary part of the frequency-dependent vortex mass $m_{v}(E)$ as a function of the energy in units of $J$ for the four different interaction strengths. The irregular frequency dependence at low frequencies in some plots is simply a reflection of incomplete broadening of our discrete Bogoliubov excitation spectrum and is without physical significance.

model adiabatic approximation and analytic continuum-model adiabatic approximation give similar values for the vortex cyclotron frequency.

\section{Vortex cyclotron motion}

In Fig. 9, we show the behavior of the real and imaginary parts of the frequency dependence in $m_{v}^{x x}=m_{v}(E=\hbar \omega)$ for various values of $U / J$. The $\omega \rightarrow 0$ limit of $m_{v}(E=\hbar \omega)$ is the adiabatic vortex mass discussed above. The contribution of particular environment Bogoliubov modes to the vortex mass increases in magnitude as $\hbar \omega$ approaches the energy of that mode from below and then changes sign at higher frequencies. The mass contribution vanishes for $\omega$ much larger than environment mode frequencies.

The real and imaginary parts of the vortex mass are related by a standard Kronig-Kramers relationship [see Eq. (34)]. We see in Fig. 9 that for the weak-interaction cases the imaginary part of the vortex mass approaches a constant at small frequencies, as in the continuum case, and that the real part of the vortex mass has a corresponding logarithmic peak at low frequencies. At strong interactions the long-wavelength low-energy contribution to $\operatorname{Im}\left[m_{v}(E=\hbar \omega)\right]$ is not dominant but a clear peak appears at the energy of the van-Hove singularity in the Bogoliubov spectrum. This peak becomes quite strong for strong interactions and the real part of the vortex mass correspondingly becomes quite large before changing sign near this frequency. Because the imaginary part of the vortex mass is relatively small and the real part is relatively frequency independent over a broad frequency regime, we conclude that the adiabatic approximation might be reasonable in describing the lattice-pinned vortex degree of freedom in strongly interacting optical lattices. Of course, as we have already explained, continuum-model estimates 
(a)

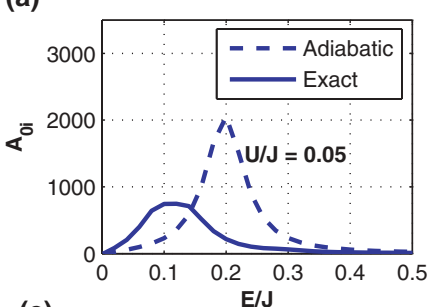

(c)

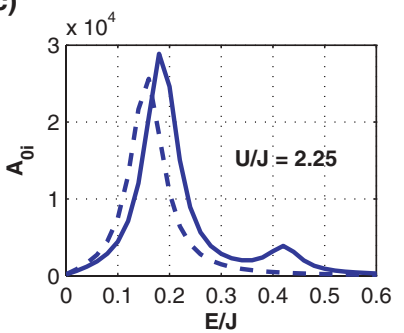

(b)

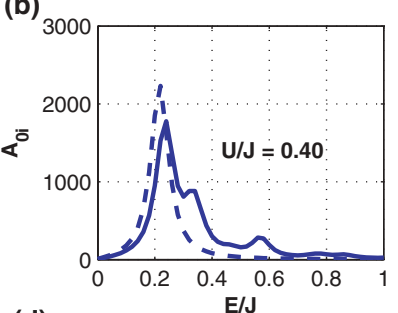

(d)

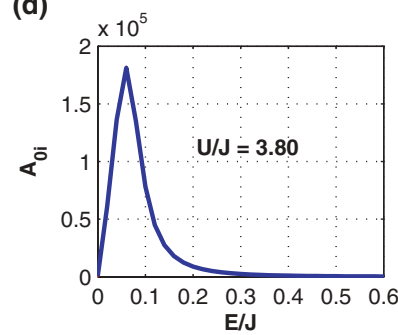

FIG. 10. (Color online) Imaginary part of vortex positionposition correlation function $A_{0 i}[\omega]$ with (dashed lines) and without (solid lines) the adiabatic approximation. The adiabatic approximation becomes more accurate when interactions are very strong (d) because the lattice-weakened Berry-phase coupling $\Gamma_{x y}$ suppresses the resonance frequency. The adiabatic approximation result for $U / J=3.80$ coincides closely with its nonadiabatic counterpart.

completely fail to describe the frequency of the mode in this regime.

In Fig. 10, we test for the existence of a sharp cyclotron mode by examining the frequency dependence of the imaginary part of the vortex position-position spectral function $A_{0 i}[\omega]=-\frac{1}{\pi} \operatorname{Im} G_{0 i}^{R}$, where $G_{0 i}^{R}=\left\langle x_{v}(\omega) x_{v}(-\omega)\right\rangle=$ $\left\langle y_{v}(\omega) y_{v}(-\omega)\right\rangle$. The corresponding adiabatic approximation in the lattice model is obtained by setting the off-diagonal elements between environmental modes $\Gamma_{\alpha, \alpha^{\prime}}$ to zero in our calculations. As expected, the adiabatic vortex picture is reasonably accurate for the strongly interacting cases. For the weak-interaction case illustrated in Fig. 9(a), the vortex cyclotron mode frequency is renormalized and is strongly broadened because $\operatorname{Im}\left[m_{v}(E)\right]$ is large at the resonance position. The adiabatic cyclotron modes in Figs. 9(b) and 9(c) have a complicated frequency dependence because of the combined influence of long-wavelength and van-Hove singularity environment modes. In Figs. 9(d), the high-frequency modes near the van-Hove singularity are strongly dominant and corrections to the adiabatic approximation are weak.

\section{CONCLUSIONS AND DISCUSSION}

In this article we have developed an approach to vortex dynamics in neutral Bose superfluids that is based on careful separation of zero modes corresponding to vortex translation from the other fluctuations around the mean-field Bose condensate. Integrating out other fluctuations leads to a vortex action with a frequency-dependent vortex mass. In the adiabatic limit, in which frequency dependence is ignored, we recover the result of Duan [29] that the vortex mass diverges logarithmically with system size in a continuum model of the superfluid. The frequency dependence of the effective mass found in our approach is similar to the result obtained by

Arovas and Freire [30] with a completely different method. The frequency-dependent effective mass has a nonzero imaginary part which captures the dissipation associated with vortex motion.

In our approach, Berry-phase coupling between orthogonal vortex displacements, which causes the vortex to behave like a charged particle in an effective magnetic field, can be calculated straightforwardly. In the adiabatic limit the vortex undergoes undamped cyclotron motion which quantummechanically leads to sharp vortex-Landau levels. Using our analytic results for the frequency-dependent complex effective mass, we conclude that in the continuum-limit vortex-Landau level physics can occur only when the system size is exponentially large compared to the vortex core size. In the case of many vortices, the vortex separation would also need to be exponentially large compared to the average distance between vortices. For these reasons it seems impossible to achieve sharp vortex-Landau levels in an atom cloud unless interaction and kinetic energies are manipulated by an optical-lattice potential.

In order to explore vortex quantum mechanics in an opticallattice system, and as a test of our analytic continuum-model results, we have repeated our calculation for an optical-lattice model in which we are able to integrate out condensate fluctuations numerically. Because of practical numerical restrictions we are able to perform calculations only for a system with a finite number ( 2000) of lattice sites. Since the continuum model is expected to apply when the vortex core size is much larger than a lattice constant, we are not fully able to simulate the regime of large isolated vortices for which we are able to obtain approximate results analytically. Nevertheless, for moderately weak interactions there is approximate agreement between our numerical results and analytic estimates based on continuum-model calculations. As interactions strengthen we find that numerical results for the adiabatic vortex mass continue to compare favorably with our analytical results even when the vortex core is smaller than an optical-lattice unit cell. The discrepancy between continuum-model estimates and numerical results is much larger for the Berry-phase coupling between orthogonal vortex displacements, that is, for the effective magnetic field responsible for vortex-Landau levels. Analytic estimates of this effective magnetic field are, in fact, not easily recovered numerically. This is because, depending on the vortex core size, either the finite size of the simulation cell cannot be ignored (for large vortex cores) or lattice pinning is important (for small vortices). In the strong-interaction limit we find that the effective magnetic field is substantially reduced, lowering the Landau level frequency and weakening its dissipative coupling to environmental modes of the condensate. According to our calculations, strong interactions enhance the opportunity to observe well-defined cyclotron motion for this unexpected reason. Of course, the collective degree of freedom in this limit is not a simple vortex displacement because of lattice-pinning effects. In addition, because the atom density modulation associated with vortex motion is weaker at strong interactions, it may be more difficult to couple to this collective motion.

Although our approach treats the environmental fluctuations within a Gaussian approximation, which is, strictly speaking, only valid for weakly interacting systems, we believe that our conclusions are more general on a qualitative level. 
This is because many of the results we find are associated with the gapless Bogoliubov dispersion of the environmental modes. This gaplessness is a result of symmetry and is preserved for strong interactions.

Finally, we remark that our approach is, in principle, general and can also be used to study the dissipative dynamics of other types of topological excitations, for example, skyrmions and monopoles in spinor Bose condensates.

\section{ACKNOWLEDGMENTS}

This work was supported by the National Science Foundation under Grant No. DMR-0606489 and by the Welch Foundation. Joseph Wang thanks Jairo Sinova and Q. Niu for helpful discussions at the early stages of this project. He also thanks Yi-Hsiang Yu for sharing his knowledge of finite-difference methods.
[1] L. Onsager, Nuovo Cimento Suppl. 6, 279 (1949).

[2] R. P. Feynman in Progress in Low Temperature Physics, edited by C. J. Gorter (North-Holland, Amsterdam, 1955), Vol. 1, p. 17.

[3] M. H. Anderson, J. R. Ensher, M. R. Matthews, C. E. Wieman, and E. A. Cornell, Science 269, 198 (1995).

[4] K. B. Davis, M.-O. Mewes, M. R. Andrews, N. J. van Druten, D. S. Durfee, D. M. Kurn, and W. Ketterle, Phys. Rev. Lett. 75, 3969 (1995).

[5] A. J. Leggett, Rev. Mod. Phys. 73, 307 (2001).

[6] F. Dalfovo, S. Giorgini, L. P. Pitaevskii, and S. Stringari, Rev. Mod. Phys. 71, 463 (1999).

[7] M. Greiner, O. Mandel, T. Esslinger, T. W. Haensch, and I. Bloch, Nature (London) 415, 39 (2002).

[8] O. Morsch and M. Oberthaler, Rev. Mod. Phys. 78, 179 (2006).

[9] K. W. Madison, F. Chevy, W. Wohlleben, and J. Dalibard, Phys. Rev. Lett. 84, 806 (2000).

[10] J. R. Abo-Shaeer, C. Raman, J. M. Vogels, and W. Ketterle, Science 292, 476 (2001).

[11] P. C. Haljan, I. Coddington, P. Engels, and E. A. Cornell, Phys. Rev. Lett. 87, 210403 (2001).

[12] E. Hodby, G. Hechenblaikner, S. A. Hopkins, O. M. Maragò, and C. J. Foot, Phys. Rev. Lett. 88, 010405 (2001).

[13] N. R. Cooper, N. K. Wilkin, and J. M. F. Gunn, Phys. Rev. Lett. 87, 120405 (2001); T.-L. Ho, ibid. 87, 060403 (2001); J. Sinova, C. B. Hanna, and A. H. MacDonald, ibid. 89, 030403 (2002).

[14] B. Paredes, P. Fedichev, J. I. Cirac, and P. Zoller, Phys. Rev. Lett. 87, 010402 (2001); N. K. Wilkin and J. M. F. Gunn, ibid. 84, 6 (2000); N. Regnault and Th. Jolicoeur, ibid. 91, 030402 (2003).

[15] N. R. Cooper, Adv. Phys. 57, 539 (2008).

[16] U. R. Fischer, P. O. Fedichev, and A. Recati, J. Phys. B 37, S301 (2004).
[17] A. A. Burkov and E. Demler, Phys. Rev. Lett. 96, 180406 (2006).

[18] P. Ao, e-print arXiv:cond-mat/0504222.

[19] N. B. Kopnin, Vortex Dynamics, part IV, in Theory of Nonequilibrium Superconductivity (Clarendon Press, Oxford, UK, 2001).

[20] P. Ao and X.-M. Zhu, Phys. Rev. B 60, 6850 (1999).

[21] Q. Niu, P. Ao, and D. J. Thouless, Phys. Rev. Lett. 72, 1706 (1994).

[22] M. Flaig, U. R. Fischer, Phys. Rev. B 74, 224503 (2006).

[23] E. B. Sonin, in Vortices in Unconventional Superconductors and Superfluids, edited by R. P. Huebener, N. Schopohl, and G. E. Volovik (Springer-Verlag, Berlin, 2002), p. 119.

[24] P. Ao and D. J. Thouless, Phys. Rev. Lett. 70, 2158 (1993).

[25] P. Nozieres and J. Vinen, Philos. Mag. 14, 667 (1966).

[26] C. Dasgupta and B. I. Halperin, Phys. Rev. Lett. 47, 1556 (1981); D. R. Nelson, ibid. 60, 1973 (1988); M. P. A. Fisher and D.-H. Lee, Phys. Rev. B 39, 2756 (1989); E. Simanek, Inhomogeneous Superconductors: Granular and Quantum Effects (Oxford University Press, London, 1994).

[27] M. P. A. Fisher, Phys. Rev. Lett. 65, 923 (1990).

[28] R. J. Donnelly, Quantized Vortices in Helium II (Cambridge University Press, Cambridge, UK, 1991).

[29] J. M. Duan, Phys. Rev. B 49, 12381 (1994).

[30] D. P. Arovas and J. A. Freire, Phys. Rev. B 55, 1068 (1997).

[31] D. J. Thouless and J. R. Anglin, Phys. Rev. Lett. 99, 105301 (2007).

[32] J. L. Gervais and B. Sakita, Phys. Rev. D 11, 2943 (1975).

[33] H. B. Braun and D. Loss, Phys. Rev. B 53, 3237 (1996).

[34] M. P. A. Fisher, P. B. Weichman, G. Grinstein, and D. S. Fisher, Phys. Rev. B 40, 546 (1989); D. van Oosten, P. van der Straten, and H. T. C. Stoof, Phys. Rev. A 63, 053601 (2001).

[35] N. H. Lindner, A. Auerbach, and D. P. Arovas, Phys. Rev. Lett. 102, 070403 (2009). 